\title{
Congenital diaphragmatic defects that present late
}

\author{
P S MALONE, A J BRAIN, E M KIELY, AND L SPITZ \\ The Hospital for Sick Children, London
}

SUMmaRY Twenty two patients (age range: 1 month to 11 years) were treated for congenital diaphragmatic defects (excluding hiatus hernia) in the six year period 1983-8. Presenting features were failure to thrive $(n=7)$, abdominal pain and vomiting $(n=4)$, chronic respiratory symptoms $(n=3)$, and inability to wean from ventilatory support $(n=3)$. The defect was an incidental finding in five patients. Operative repair was performed with no mortality or serious postoperative morbidity. Dramatic improvement occurred in 15 of the 17 symptomatic patients. Awareness of the differential diagnosis should avoid delay in diagnosis or inappropriate treatment. Surgical correction is strongly recommended in all cases.

Excluding hiatus hernia there are two types of congenital diaphragmatic defect-hernia and eventration. A hernia is a protrusion of the abdominal viscera through the diaphragm with or without a hernial sac. An eventration may be defined as an abnormally high leaf of an intact diaphragm as a result of paralysis, aplasia, or atrophy of the muscle fibres. Its uninterrupted continuity differentiates it from a hernia. ${ }^{1}$

Between $5 \%$ and $30 \%$ of congenital defects present after the neonatal period, ${ }^{12}$ but despite this high incidence congenital diaphragmatic defects are not often recognised. This results in diagnostic delay and inappropriate treatment, both of which may be fatal. ${ }^{3-5}$ Most reports refer only to true diaphragmatic hernias and exclude eventration. As both present in a similar manner and cannot always be differentiated before operation, we believe that eventrations should be included. The purpose of this paper is to draw attention to these defects and the circumstances leading to diagnostic delay and inappropriate treatment.

\section{Patients and methods}

The records of patients over 1 month of age with congenital diaphragmatic defects treated in this unit from 1983 to 1988 were retrospectively reviewed. Patients with acquired lesions were excluded. There were 22 patients (13 girls and nine boys) who represent $13 \%$ of all the congenital diaphragmatic defects treated during this period. Age at operation ranged from 1 month to 11 years (median 9 months).
There were 18 hernias and four eventrations (table 1). Associated abnormalities included midgut malrotation $(n=8)$ and the Prader-Willi syndrome with Langerhans cell histiocytosis $(n=1)$. No other associated congenital anomalies were noted, but four patients had group B $\beta$ haemolytic streptococcal infection.

Presenting features are listed in table 2. Three of the seven patients who failed to thrive also had

Table 1 Types of defects

\begin{tabular}{lllll}
\hline & $\begin{array}{l}\text { Sac } \\
\text { present }\end{array}$ & Left & Right & Bilateral \\
\hline $\begin{array}{l}\text { Bochdalek hernia } \\
(n=10)\end{array}$ & 5 & 8 & 2 & 0 \\
$\begin{array}{c}\text { Central hernia } \\
(n=5)\end{array}$ & 2 & 2 & 3 & 0 \\
$\begin{array}{l}\text { Morgagni hernia } \\
(n=3)\end{array}$ & 1 & 0 & 3 & 0 \\
$\begin{array}{c}\text { Eventration (n=4) } \\
\text { Not }\end{array}$ & 0 & 3 & 1 \\
\hline
\end{tabular}

Table 2 Presenting features

\begin{tabular}{ll}
\hline & No of infants \\
\hline Failure to thrive & 7 \\
Abdominal pain and vomiting & 4 \\
Chronic respiratory symptoms & 3 \\
Inability to breath without ventilator & 3 \\
No symptoms (incidential finding) & 5 \\
\hline Total & 22 \\
\hline
\end{tabular}


dyspnoea during feeding. All three patients who were dependent on ventilatory support were premature and required ventilation soon after birth for primary lung disease. The defects were all right sided, and a pleural effusion was erroneously diagnosed and chest drain inserted in each case. One of these patients suffered a catastrophic haemorrhage as a result of trauma to the intrathoracic liver necessitating emergency thoracotomy. The five patients in whom the defect was an incidental finding presented with symptoms unrelated to the diaphragmatic defect. Three had upper respiratory tract infections, one had otitis media, and one had Langerhans cell histiocytosis. The clinical impression of dextrocardia was the indication for chest radiograph in three patients. A routine 'staging' radiograph was carried out in the patient with histiocytosis, but the indication for radiography in the remaining patient was not clear.

Diagnostic confusion occurred in three patients, two of whom had abnormal radiographs at the referring hospitals but normal ones on admission to this unit. Subsequent pictures showed the defect in one patient, while in the remaining patient a hernia was confirmed at operation. One patient with an eventration had an initial normal chest radiograph, followed by radiographs showing the raised hemidiaphragm. As the initial picture was normal the correct diagnosis was not immediately considered. In these patients diaphragmatic screening and ultrasound scans were helpful in confirming the diagnosis.

All patients underwent surgical repair. The approach was through a laparotomy in 14, a thoracotomy in six, and a thoracoabdominal incision in two. The defect was sutured directly in 15 and patched with Dacron in three; the four eventrations were plicated. The eight patients with malrotation had a simultaneous Ladd's procedure. Postoperative chest drains were not used in 14 patients; two subsequently required one, one for a suspected tension pneumothorax (that was not confirmed) and one for a persistent pleural effusion.

\section{Results}

There were no serious postoperative complications and no deaths. Follow up ranged from 3 months to 3.5 years (median 6 months). Five patients who had failed to thrive improved immediately with weight gain increasing from below the third centile to above the 10th centile within two months of operation. The remaining two patients who had failed to thrive seemed to received no benefit. The patients with chronic respiratory and gastrointestinal symptoms all improved rapidly after operation. The three patients who had been dependent on ventilatory support were all successfully extubated within a week of operation.

\section{Discussion}

This series emphasises the varied presentation of congenital diaphragmatic defects diagnosed after the neonatal period. Chronic respiratory and gastrointestinal symptoms have been previously described. ${ }^{12}$ Less than half of the patients in our series, however, had these symptoms. Failure to thrive was the commonest presentation, unlike the series described by Berman et al in which only one patient presented with failure to thrive. ${ }^{2}$ This report is the first to our knowledge to emphasise that poor weight gain is a common presenting feature of congenital diphragmatic defects diagnosed after the neonatal period.

The association of group B $\beta$ haemolytic streptococcal infection and late presenting diaphragmatic hernia (as occurred in four of our patients) has been previously described..$^{7-9}$ Graviss et al stated that the group $B$ infection is not causally related to the development of a diaphragmatic hernia. ${ }^{7} \mathrm{We}$ postulate that compression of the lung with resultant consolidation may predispose the infant to this infection.

One of the factors contributing to diagnostic delay is a normal chest radiograph. Wiseman and MacPherson in 1977 coined the term 'acquired' congenital diaphragmatic hernia for lesions presenting at variable times after documented normal chest radiographs. ${ }^{6}$ This contributed to the diagnostic delay in one of our patients. Two patients had abnormal chest radiographs at the referring hospital, but that on admission to this unit was normal. It would seem that the herniated abdominal contents can reduce spontaneously through the defect. The presence of a normal chest radiograph after one showing a diaphragmatic defect, therefore, does not preclude the diagnosis.

The risks of misdiagnosing right sided congenital diaphragmatic hernias as persistent pleural effusions in patients who cannot be weaned from a ventilator are stressed. ${ }^{47}$ Various methods have been recommended to evaluate the integrity of the diaphragm. These include radionucleatide scanning of the liver and spleen, umbilical venography, computed tomography, contrast peritoneography, barium meal examination, diaphragmatic screening, and ultrasonography. Diaphragmatic screening is useful only in patients who are breathing spontaneously. Ultrasound scans and barium meal examinations are helpful in ventilated infants. A diaphragmatic defect should be suspected in every patient in whom the diaphragm is not clearly visible on radiography. 
Further investigations aimed at establishing the presence of an intact diaphragm should therefore be undertaken. Keeping the possibility of the diagnosis in mind should avoid undue delay, misdiagnosis, and inappropriate treatment.

Three patients $(23 \%)$ had Morgagni hernias. This is a higher incidence than the usually reported $1-2 \%$ of all congenital diaphragmatic hernias and reflects the fact that they can be small defects that are not usually detected at birth. ${ }^{10}$ Booker et al reported a similarly high incidence of Morgagni hernias in older children. ${ }^{3}$ The defects are usually right sided, as in our three cases, and lie anteriorly between the xiphisternum and costal muscle fibres of the diaphragm. Two of our patients had gastrointestinal symptoms (intermittent colicky abdominal pain and vomiting) and their hernias contained colon. The small defect increases the risks of strangulation, and in the two cases reported by Booker et al necrotic bowel was found. ${ }^{3}$

Once the diagnosis of a diaphragmatic defect has been established, surgical correction should be performed without delay. We plan operations for the next available routine list, which was usually a matter of days. Even with this policy one of our patients developed intestinal obstruction while waiting and required an emergency operation. We therefore recommend that all patients stay in hospital before operation, but emergency operation is not normally indicated. Other complications that have developed before operation include cardiorespiratory arrest ${ }^{2}$ and intestinal infarction. ${ }^{35}$

Our approach to these late diagnosed diaphragmatic defects is as follows: a barium meal and follow through examination is carried out to exclude the presence of a malrotation. In patients without a malrotation we recommend a transthroacic approach to the repair of the defect. Patients with a malrotation require an abdominal approach to facilitate a simultaneous Ladd's procedure.

\section{References}

1 Bonham Carter RE, Waterston DJ, Aberdeen E. Hernia and eventration of the diaphragm in childhood. Lancet 1962;i:656-9.

2 Berman L, Stringer D, Ein SH, et al. The late presenting pediatric Bochdalek hernia: a 20 year review. J Pediatr Surg 1988;23:735-9.

3 Booker PD, Meerstadt PWD, Bush GH. Congenital diaphragmatic hernia in the older child. Arch Dis Child 1981;56:253-7.

${ }^{4}$ Chilton HW, Chang HT, Jones MD, et al. Right-sided congenital diaphragmatic herniae presenting as pleural effusions in the newborn: dangers and pitfalls. Arch Dis Child 1978;53:600-3.

5 Wooley MM. Delayed appearance of a left posterolateral diaphragmatic hernia resulting in significant small bowel necrosis. J Pediatr Surg 1977;12:673-4.

6 Wiseman NE, MacPherson RI. 'Acquired' congenital diaphragmatic hernia. J Pediatr Surg 1977;12:657-65.

7 Graviss ER, Danis RK, Lewis JE, et al. Peritoneography diagnosis of delayed onset right-sided diaphragmatic hernias masquerading as pleural effusion. J Pediatr 1980;97:119-22.

8 Young LW, McClead RE, Graham M, et al. Radiological case of the month: postnatal appearance of diaphragmatic hernia. Am J Dis Child 1978;139:1137-8.

9 McCarten KM, Rosenberg HK, Borden S, et al. Delayed onset of right diaphragmatic hernia associated with group B streptococcal infection in the newborn. Radiology 1981;139:385-9.

${ }^{10}$ Synder WH, Greaney EM. Congenital diaphragmatic hernia: 77 consecutive cases. Surgery 1965;57:576-88.

Correspondence to Mr PS Malone, Department of Paediatric Surgery, The Hospital for Sick Children, Great Ormond Street, London WC1N 3JH.

Accepted 29 June 1989 